\title{
MONITORING STRATEGIES OF ENTERPRISE'S EMISSION REDUCTION WITH ASYMMETRIC INFORMATION
}

\author{
Chen Kegui ${ }^{1}$, Wang Xinyu ${ }^{1, *}$, Huang Min $^{2}$ and Ren Liang ${ }^{3}$
}

\begin{abstract}
More recently, the Chinese Government had been making appreciable efforts to stimulate and encourage entrepreneurial emission reductions. These efforts could be boosted by availability of dual asymmetric information about the adopted technology for efficient reduction and enterprises' investment strategies, possibly leading to adverse selection and moral hazard. This paper incorporates Stackelberg game model and incentive mechanism theory to find a feasible solution to dual asymmetric information problem. Hence firstly, through application of linear function of the total emission, an emission reduction contract would be formulated for regulating incentive payments from government to supervisory authorities followed by derivation of a two stage principal-agent monitoring model based on the enterprise's private information and action respectively. Some findings have been obtained through analysis of the monitoring models along with their comparison with the results of no monitoring scenario which confirm the effectiveness of hiring the supervisory authorities for monitoring of enterprises' emission reduction, which, ultimately, would facilitate increasing payoff and efficient emission reduction. In this perspective, the action-based monitoring mechanism depicts overall government dominance. Several, managerial insights have also been provided for various scenarios and propositions and all the findings are illustrated numerically. It could prove highly beneficial and significant to the government in encouraging entrepreneurial emission reductions.
\end{abstract}

Mathematics Subject Classification. 91A80, 91B24, 91B26.

Received April 25, 2020. Accepted September 2, 2020.

\section{INTRODUCTION}

A substantial concentration of the Greenhouse gases on the Earth has instigated increasing global warming. Hence in near future, the resultant climatic changes would pose serious social and environment challenges. As far as the global warming issues are concerned, all the researchers across the globe have unanimously stressed upon the need of taking stringent actions to effectively curb carbon emissions (see [16]). In this regard, a series of global climate summits concerning emission-related issues have been held since the 1990s which indicates that the problem of emission reduction had been given unprecedented global importance. United Nations Framework Convention on Climate Change (UNFCCC) is the first international covenant on controlling greenhouse gases

Keywords. Overconfidence, effort-dependent demand, delegation, salesforce compensation.

1 School of Management, China University of Mining and Technology, Xuzhou, P.R. China.

2 College of Information Science and Engineering, Northeastern University, Shenyang, P.R. China.

3 School of Management, Wuhan University of Science and Technology, Wuhan, P.R. China.

*Corresponding author: wangxinyu@cumt.edu.cn 
emission (see [8]). Presently, many countries including China are faced with the dilemma of deciding between economic growth and environmental deterioration. More recently, Intergovernmental Panel on Climate Change (IPCC) had reported an unprecedented increase in global emissions levels of greenhouse gases. Many governments and organizations have adopted mechanisms or enacted legislations to control carbon emissions. According to the European Commission surveys, $83 \%$ of Europeans are highly attuned to environmental impacts, mainly the carbon footprint, while procuring products of daily use (see [4]). In this regard, the government interest plays a vital role in emission reduction because enterprises are risk-averse and interest-driven, and absence of the corresponding intervention and incentives policies prompts relatively weak entrepreneurial interest in emission reduction. Hence, enterprise's reduction needs to be monitored, controlled and guided by the government. The enterprises have access to superior information about emissions and required reduction mechanisms, the necessary technology required, the corresponding cost, requirement of capital investment, emission reduction performance and expected revenue. In the process of enterprise's implementation of emission reduction policies, there exists asymmetric information between the government and enterprises.

Under the national policy norms for emission reduction, the enterprise might instigate adverse selection problem and moral hazard. In some cases, enterprises increase environmental pollution while simultaneously obtaining government's subsidies and in event of inspection/supervision temporarily operate devices acquired for emission reduction while others tend to exaggerate their investment figures, capacity and technology level while reporting the emission reduction statistics to the concerned authorities. The presence of asymmetric information between government and the enterprises, causes latter great inconvenience while formulating/implementing emission reduction policies. Hence, for the effective use of the marketing mechanisms, reducing information asymmetry between government and enterprises, improving efficiency of government regulatory policies, and inducing enterprises' emission reduction, it is necessary to develop an understanding regarding formulation of effective incentive contracts by the government, and further study the joint effect of asymmetric information and supervisory strategy on the emission reduction.

Monitoring and development of incentive contracts are yet another effective way of combating shirking and asymmetric information (see $[1,2,10,12])$. In spite of global adoption of monitoring mechanisms, relatively scarce knowledge is available to supervise the enterprise's emission reduction under dual asymmetric information. As far as impact of monitoring on emission reduction is concerned, this paper represents monitoring as an activity which provides information on enterprise's technological investment for facilitating reduction. The modeling choices have been guided by the principal-agent and monitoring strategy. This study is principally aimed at presence of asymmetric information and action and hence focuses on effectiveness of two different monitoring mechanisms, i.e., information-based and action-based in facilitating environmental regulation. Special focus had been made on addressing the following issues: (1) Should the government directly sign monitoring/incentive contracts with the enterprise or involve supervisory authorities for effective monitoring of the enterprise? (2) What is the impact of monitoring mechanisms on incentive contracts? (3) Of the two monitoring mechanisms, which one is mutually beneficial to both the government and the enterprise? To address these issues, firstly, a no monitoring scenario is presented as the benchmark case, in which the government directly signs the contract with the enterprise while in another case, the government hires a professional authority to monitor the enterprise's unobservable technology and investment information to facilitate effective reduction finally, followed by derivation of respective monitoring/incentive contracts and comparison of two monitoring mechanisms with the benchmark case.

The rest of the paper has been organized as follows: Section 2, gives a brief overview of the literature consulted while formulating this study. Section 3 discusses problem statement and model assumption while Section 4 discusses the optimal terms of contracts for the no monitoring scenario as a benchmark model, and further elaboration/discussion of optimal contracts for the two monitoring mechanisms, in Section 5. Section 6, compares the results of the two monitoring mechanisms and studies the effectiveness of monitoring mechanisms, and demonstrates a numerical study. Finally, followed by conclusions and directions for future research in Section 7. 


\section{Literature REVIEW}

This paper is primarily related to two strands of literature; i.e., the research on low-carbon and emission reduction management and the economic literature on agency and monitoring mechanisms which would be discussed below.

Besides pure incentive contracting (see [11]), monitoring and associated incentive contracts are another way to combat entrepreneurial non-compliance (see $[1,2,10,12]$ ). Joseph and Thevaranjian [12] analyzed a framework that simultaneously examines the role of both monitoring and incentives in the design of sales force control systems. Demougin and Fluet [7] analyzed the trade-off between monitoring and incentives in a principal-agent relationship with moral hazard. Huddart and Liang [10] had studied the profit sharing and monitoring in partnerships, they considered partnerships among risk-averse professionals endowed with a risky and personally costly production technology and a personally costly monitoring technology providing contractible noisy signals about partners' productive efforts. Every partner performs the same tasks and has the same characteristics. The authors illustrated partners' productive and monitoring efforts under different monitoring mechanisms. All of the above monitoring mechanisms are only based on moral hazard. Yang et al. [22] studied two monitoring mechanisms based on the uncertainty theory during new product development: the ideal information-based mechanism and the effort-based monitoring mechanism. In order to determine the impact of monitoring mechanisms on incentive contracts and existence of a dominant monitoring mechanism, he presented two bi-level principal-agent monitoring models along with derivation of their respective optimal incentive contracts. Kung and Chen [13] investigated a multilayer supply chain approach involving two types of resellers: a knowledgeable reseller who observes the market condition, and a diligent reseller who monitors the service level, it was observed that delegation to a diligent reseller enhances information acquisition. The results also highlighted the relative importance of adverse selection and moral hazard. To facilitate effective supervision/monitoring of emission reduction, Mackenzie and Ohndorf [17] extended the general framework on incomplete enforcement of policy instruments to reflect the particularities of credit-based mechanisms under asymmetric information. The results indicated that, depending on the actual abatement cost and penalty schemes, optimal monitoring for credit-based systems is often discontinuous and differs significantly from those applied for cap-and-trade schemes or environmental taxes. Sheng et al. [19] showed that a system capable of simultaneous monitoring, reporting and verification could facilitate achievement of optimum social emission level. Wu et al. [20] applied evolutionary game theory to build an evolutionary model of low-carbon strategies involving a complex network of enterprises from perspective of government-enterprise game. This article adopts a monitoring strategy to solve the enterprise's moral hazard and adverse selection problem and further analyze its effectiveness in comparison with the two existing monitoring strategies to determine a dominant monitoring strategy.

The main contributions of this paper are three-fold. Firstly, due to presence of dual asymmetric information, information-based and action-based monitoring mechanisms have been proposed for the government to induce emission reduction. This is followed by an in-depth analysis of their influence on decisions making ability of the stake holders. Secondly, non-monitoring scenario is designated the benchmark case, and compared with the two monitoring mechanisms verify their validity in determining a dominant monitoring mechanism from the government's perspective. Finally, this model stresses the government to adopt action-based monitoring mechanism as a means to boost its income and further facilitate emission reduction since the enterprise no longer obtains additional information rent under information-based monitoring mechanism while increasing its investment base. Hence, the government will profit more by monitoring the enterprises, since action-based monitoring mechanism is more favorable to the government.

\section{Problem Formulation AND MOdel ASSUMPtion}

This study focuses on designing a monitoring contract for government to induce the enterprise (he) to reduce the emissions assuming that the initial emission amount $Q_{0}$ is random and depends on the capital $z$ spent on emission reduction exerted by the enterprise and technological level $k$ of enterprise's emission reduction. 
The following exponential function is adopted to model the enterprise's final emission expressed as: $Q=Q_{0} \varepsilon e^{-k z}$, where $\varepsilon$ is a distributed random variable with mean $\mu$ and variance $\sigma^{2}$, and $\varepsilon$ is independent of $Q_{0}, z$ and $k$, thus the enterprise's actual emission reduction amount is $Q_{0}\left(1-\varepsilon e^{-k z}\right)$.

The enterprise has access to superior information, along with the investment level $z$ for reduction. The technological level $k$ is also his private information, whereas, the government treats $k \in[\underline{k}, \bar{k}]$ as random with distribution $F(k)$ and density $f(k)$, where $\underline{k}>0, F(\underline{k})=0$ and $F(\bar{k})=1$ hold. We further assume that $F(k)$ satisfies the Increasing Failure Rate property (IFR), i.e., both the inverse failure rate $H(k)=\bar{F}(k) / f(k)$ and $h(k)=\bar{F}(k) / k f(k)$ are increasing in $k$, where $\bar{F}(k)=1-F(k)$, obviously, $H(k), h(k) \geq 0$ (see [13]).

Since the government expects maximum emission reductions, this paper follows the modeling assumptions of the emission-cost trade-off literature and assumes that the government's emission reduction revenue or bonuses depend linearly on emissions amount, i.e., $\pi(Q)=B-\delta Q$, where $\delta>0$ is the government's emission reduction profit margin, and it measures the superior government's attention levels for emission reduction.

The government should hire professional authorities to monitor the enterprise's investment/ technological level for emission reduction $k$, so the government can hire the professional supervisory authorities to monitor the enterprise's investment and technology level for reduction. In our monitoring model, the government contracts with the supervisory authorities and then the supervisory authorities contracts with the enterprise are based on the realized amount of emission. We restrict our attention to the class of linear contracts because of the prevalence in practice. Specifically, we use a linear compensation contract $(\alpha, \beta)$ to denote the contract signed between the supervisory authorities and the enterprise, where $\alpha$ is the fixed payment and $\beta$ is the commission rate. According to this contract, the enterprise receives an aggregate payment $s(Q)=\alpha-\beta Q$ if the actual amount of emission $Q$ is realized. Therefore, the enterprise's net profit function could be expressed as:

$$
\pi_{E}=s(Q)-z=\alpha-\beta Q-z .
$$

Similarly, if the government and the supervisory authorities sign the contract $(u, v)$ with fixed payment $u$ and the commission rate $v \geq 0$, the supervisory authorities will receive $t(Q)=u-v Q$ from the government and the resultant net payoff is given by

$$
\pi_{M}=t(Q)-s(Q)=u-\alpha-(v-\beta) Q,
$$

and the government's expected profit function could be expressed as

$$
\pi_{G}=\pi(Q)-t(Q)=B-u+(v-\delta) Q .
$$

To capture the different risk preference of the three parties, we assume that the government and the supervisory authorities are risk-neutral, i.e., their objective are to maximize their expected profit, while the enterprise is risk-averse and maximizes his expected utility. Here, the mean-standard deviation method is introduced to measure the enterprise's risk concerns. According to the literature (see $[5,9,14,16,21]$ ), the expected mean-standard deviation utility of the enterprise is

$$
U_{E}=\mathrm{E}\left(\pi_{E}\right)-\rho \sqrt{\operatorname{Var}\left(\pi_{E}\right)},
$$

where $\rho$ denotes the risk-averse degree of the enterprise, $\rho>0$, and $\rho$ is common knowledge. $\mathrm{E}\left(\pi_{E}\right)$ and $\operatorname{Var}\left(\pi_{E}\right)$ are the mean and the variance of the enterprise's profit $\pi_{E}$ respectively. Let $\underline{\pi}$ be the utility associated with the enterprise's external opportunities, i.e., the corresponding reservation utility level. The reservation utility of supervisory authorities $I$ and $A$ are assumed to be $\underline{I}$ and $\underline{A}$ respectively. Thus, the enterprise's actual expected utility would be

$$
U_{E}=\alpha-(\mu+\rho \sigma) Q_{0} \beta e^{-k z}-z,
$$

where $k Q_{0}(\mu+\rho \sigma) \beta \geq 1$ holds, i.e., $Q_{0} \geq \frac{1}{k(\mu+\rho \sigma) \beta}$.

Here the government, enterprise and the supervisory authorities could all be assumed completely rational, and tend to maximize their own utility level. At first no monitoring scenario would be investigated as the benchmark 
case, wherein the government directly signs the contracts with the enterprise without the participation of the supervisory authorities. Furthermore, two monitoring mechanisms would be considered: the information-based and the action-based, followed by derivation of their respective optimal incentive contracts. Finally, examine how effective are the monitoring mechanisms and investigate the existence of a dominative monitoring mechanism. The above assumptions are mutual to all the parties concerned. For notational convenience, we use $\mathrm{E}[\cdot]$ and $\mathrm{E}_{k}[\cdot]$ to represent the mathematical expectation. The subscripts "I", "A", "G", "M" and "E", respectively, denote monitoring Information, monitoring Action, Government, the Monitoring authorities and the Enterprises, respectively, and the superscript "*" denotes the optimal cases.

\section{Benchmark Without MONITORING}

Firstly, no monitoring scenario is considered as a benchmark case in which the government directly signs the contracts with the enterprise. In this government-enterprise relationship, both the technology/investment level for reduction are the enterprise's private information, the government faces a conundrum of adverse selection and moral hazard problems. Furthermore, this no monitoring problem would also be compared with the two monitoring models (information-based/action-based) to illustrate the benefits of both the monitoring mechanisms.

The ultimate goal of the government is to formulate such incentive contracts which would facilitate profit maximization based on revelation principle. The sequence of events for no monitoring is as follows: (1) The enterprise's technology level is observed; (2) Due to government inability to observe the enterprise's technology level, it offers a menu of compensation contracts $(\alpha(k), \beta(k))$ for the enterprise to self-select; (3) The enterprise decides whether or not to participate and, if so, which contract to sign in the menu based on his private technology level $k ;(4)$ Under a signed contract $(\alpha(k), \beta(k))$, the enterprise determines his investment level $z(k)$ accordingly. (5) When the final emission $Q$ is realized, the government collects the emission reduction revenue, and the enterprise is compensated.

We can get the enterprise's expected utility of equation (3.5), namely

$$
U_{E}(k)=\alpha(k)-(\mu+\rho \sigma) Q_{0} \beta(k) e^{-k z}-z .
$$

Maximizing the expected utility is equivalent to choosing the optimal investment level

$$
z(k)=\arg \max _{z \geq 0}\left\{\alpha(k)-(\mu+\rho \sigma) Q_{0} \beta(k) e^{-k z}-z\right\} .
$$

Based on the first-order necessary condition

$$
\frac{\mathrm{d} U_{E}(k)}{\mathrm{d} z}=k Q_{0}(\mu+\rho \sigma) \beta(k) e^{-k z}-1=0 .
$$

Here the optimal investment level is obtained in Lemma 4.1, and it satisfies

$$
\frac{\mathrm{d}^{2} U_{E}(k)}{\mathrm{d} z^{2}}=-k^{2} Q_{0}(\mu+\rho \sigma) \beta(k) e^{-k z}<0,
$$

which implies the concavity of $U_{E}(k)$, and $z^{*}(k)$ satisfies the second-order condition.

Lemma 4.1. Under the no monitoring scenario, in view of the menu of contracts $(\alpha(k), \beta(k))$, the enterprise's optimal investment level $z^{*}(k)$ satisfies

$$
z^{*}(k)=\frac{\ln \left[k Q_{0}(\mu+\rho \sigma) \beta(k)\right]}{k} .
$$


From equation (4.5), it is evident that the optimal investment level is independent of the base salary $\alpha(k)$. Substituting this first-best investment level into equation (4.1), we can obtain

$$
U_{E}(k)=\alpha(k)-\frac{1+\ln \left[k Q_{0}(\mu+\rho \sigma) \beta(k)\right]}{k} .
$$

Substituting equation (4.5) into the government's optimal problem (3.3), we can get the government's expected profit as

$$
\mathrm{E}\left(\pi_{G}\right)=B-\alpha(k)+\frac{\mu}{k(\mu+\rho \sigma)} \frac{\beta(k)-\delta}{\beta(k)} .
$$

To characterize the optimal compensation design problem, backward induction is adopted and initiated with the enterprise's problem. Suppose the enterprise has observed $k$ but instead has chosen the contract $\left(\alpha\left(k^{\prime}\right), \beta\left(k^{\prime}\right)\right)$ related to $k^{\prime}$, then the investment level is given by

$$
z\left(k^{\prime} \mid k\right)=\frac{\ln \left[k Q_{0}(\mu+\rho \sigma) \beta\left(k^{\prime}\right)\right]}{k} .
$$

Thus, with this, the enterprise's expected utility would be

$$
U_{E}\left(k^{\prime} \mid k\right)=\alpha\left(k^{\prime}\right)-\frac{1+\ln \left[k Q_{0}(\mu+\rho \sigma) \beta\left(k^{\prime}\right)\right]}{k} .
$$

The enterprise's Individual Rationality (IR) constraint and Incentive Compatibility (IC) constraint could be expressed as follows

$$
\begin{aligned}
(\mathrm{IR}) U_{E}(k) & =\alpha(k)-\frac{1+\ln \left[k Q_{0}(\mu+\rho \sigma) \beta(k)\right]}{k} \geq \underline{\pi}, \\
(\mathrm{IC}) U_{E}(k \mid k) & =\alpha(k)-\frac{1+\ln \left[k Q_{0}(\mu+\rho \sigma) \beta(k)\right]}{k} \\
& \geq U_{E}\left(k^{\prime} \mid k\right)=\alpha\left(k^{\prime}\right)-\frac{1+\ln \left[k Q_{0}(\mu+\rho \sigma) \beta\left(k^{\prime}\right)\right]}{k} .
\end{aligned}
$$

Equation (4.11) implies

$$
\left.\frac{\mathrm{d} U_{E}\left(k^{\prime} \mid k\right)}{\mathrm{d} k^{\prime}}\right|_{k^{\prime}=k}=\alpha\left(k^{\prime}\right)-\frac{\beta\left(k^{\prime}\right)}{k \beta(k)}=0
$$

thus, we can obtain

$$
\alpha\left(k^{\prime}\right)=\frac{\beta\left(k^{\prime}\right)}{k \beta(k)} .
$$

Under the constraints (4.10) and (4.11), the government's decision problem could be transformed into

$$
\max _{\alpha(k), \beta(k)} \int_{\underline{k}}^{\bar{k}}\left[B-\alpha(k)+\frac{\mu}{k(\mu+\rho \sigma)} \frac{\beta(k)-\delta}{\beta(k)}\right] \mathrm{d} F(k) .
$$

From equations (4.11) and (4.13), we derive equation (4.15)

$$
\frac{\partial U_{E}(k)}{\partial k}=\alpha(k)+\frac{\ln \left[k Q_{0}(\mu+\rho \sigma) \beta(k)\right]}{k^{2}}-\frac{\beta\left(k^{\prime}\right)}{k \beta(k)}=\frac{\ln \left[k Q_{0}(\mu+\rho \sigma) \beta(k)\right]}{k^{2}} \geq 0 .
$$

Thus, $U_{E}(k)$ is increasing in $k$, the IR constraint (4.10) implies that $\min U_{E}(k)=U_{E}(\underline{k})=\underline{\pi}$ at the optimal solution. Consequently

$$
U_{E}(k)=U_{E}(\underline{k})+\int_{\underline{k}}^{k} \frac{\ln \left[\tau Q_{0}(\mu+\rho \sigma) \beta(\tau)\right]}{\tau^{2}} \mathrm{~d} \tau=\underline{\pi}+\int_{\underline{k}}^{k} \frac{\ln \left[\tau Q_{0}(\mu+\rho \sigma) \beta(\tau)\right]}{\tau^{2}} \mathrm{~d} \tau .
$$


To substitute the equation (4.13) into equation (4.10), and the binding IR constraint leads to

$$
\alpha(k)=\underline{\pi}+\int_{\underline{k}}^{k} \frac{\ln \left[\tau Q_{0}(\mu+\rho \sigma) \beta(\tau)\right]}{\tau^{2}} \mathrm{~d} \tau+\frac{1+\ln \left[k Q_{0}(\mu+\rho \sigma) \beta(k)\right]}{k} .
$$

Replace the $\alpha(k)$ and equation (4.5) in the objective function (4.14), the government's objective is reduced to

$$
\begin{aligned}
\max _{\beta(k)} \int_{\underline{k}}^{\bar{k}} & {\left[-\int_{\underline{k}}^{k} \frac{\ln \left[\tau Q_{0}(\mu+\rho \sigma) \beta(\tau)\right]}{\tau^{2}} \mathrm{~d} \tau-\frac{1+\ln \left[k Q_{0}(\mu+\rho \sigma) \beta(k)\right]}{k},\right.} \\
& \left.+\frac{\mu}{k(\mu+\rho \sigma)} \frac{\beta(k)-\delta}{\beta(k)}\right] \mathrm{d} F(k)+B-\underline{\pi} .
\end{aligned}
$$

By solving the problem (4.18), Proposition 4.2 is obtained, which characterizes the optimal menu contract $\left(\alpha^{*}(k), \beta^{*}(k)\right)$ designed directly by the government.

Proposition 4.2. Under the no monitoring scenario, the optimal menu of contract $\left(\alpha^{*}(k), \beta^{*}(k)\right)$ is given by

$$
\begin{aligned}
& \beta^{*}(k)=\frac{\mu \delta}{(\mu+\rho \sigma)(1+h(k))}, \\
& \alpha^{*}(k)=\underline{\pi}+\int_{\underline{k}}^{k} \frac{\ln \left[\tau Q_{0}(\mu+\rho \sigma) \beta^{*}(\tau)\right]}{\tau^{2}} \mathrm{~d} \tau+\frac{1+\ln \left[k Q_{0}(\mu+\rho \sigma) \beta^{*}(k)\right]}{k},
\end{aligned}
$$

the corresponding optimal investment level for the emission reduction is given by

$$
z^{*}(k)=\frac{\ln \left[k Q_{0}(\mu+\rho \sigma) \beta^{*}(k)\right]}{k}=\frac{\ln \left[\frac{k Q_{0} \mu \delta}{1+h(k)}\right]}{k},
$$

the enterprise's optimal expected utility is

$$
U_{E}^{*}(k)=\underline{\pi}+\int_{\underline{k}}^{k} \frac{\ln \left[\tau Q_{0}(\mu+\rho \sigma) \beta^{*}(\tau)\right]}{\tau^{2}} \mathrm{~d} \tau,
$$

with the optimal menu contracts, the government would receive an expected profit $\mathrm{E}_{k}^{*}\left(\pi_{G}\right)$ as

$$
\mathrm{E}_{k}^{*}\left(\pi_{G}\right)=B-\underline{\pi}-\frac{\rho \sigma}{k(\mu+\rho \sigma)}-\frac{1+h(k)}{k} \ln \left[\frac{k Q_{0} \mu \delta}{1+h(k)}\right]-\frac{1+h(k)}{k},
$$

where $h(k)=\frac{1-F(k)}{k f(k)}, Q_{0} \geq \frac{1}{k(\mu+\rho \sigma) \beta^{*}(k)}$.

Proof. Due to $F(\underline{k})=0, F(\bar{k})=1$, and to use the routine approach of changing the order of integration:

$$
\int_{\underline{k}}^{\bar{k}} \int_{\underline{k}}^{k} \frac{\ln \left[\tau Q_{0}(\mu+\rho \sigma) \beta(\tau)\right]}{\tau^{2}} \mathrm{~d} \tau \mathrm{d} F(k)=\int_{\underline{r}}^{\bar{r}} \frac{\ln \left[k Q_{0}(\mu+\rho \sigma) \beta(k)\right]}{k^{2}} \frac{1-F(k)}{f(k)} \mathrm{d} F(k) .
$$

The government's problem is reduced to $\max _{\beta(k)} \int_{\underline{k}}^{\bar{k}} R(\beta, k) \mathrm{d} F(k)$, where

$$
\begin{aligned}
R(\beta, k)= & B-\underline{\pi}-\frac{\ln \left[k Q_{0}(\mu+\rho \sigma) \beta(k)\right]}{k^{2}} \frac{1-F(k)}{f(k)} \\
& -\frac{1+\ln \left[k Q_{0}(\mu+\rho \sigma) \beta(k)\right]}{k}+\frac{\mu}{k(\mu+\rho \sigma)} \frac{\beta(k)-\delta}{\beta(k)} .
\end{aligned}
$$


Based on the first-order necessary condition

$$
\frac{\mathrm{d} R}{\mathrm{~d} \beta}=\frac{\frac{\mu \delta}{\mu+\rho \sigma}-(1+h(k)) \beta}{k \beta^{2}},
$$

obviously, $\frac{\mu \delta}{\mu+\rho \sigma}-(1+h(k)) \beta$ is decreasing in $\beta$, if $\beta^{*}(k)<\frac{\mu \delta}{(\mu+\rho \sigma)(1+h(k))}, \frac{\mathrm{d} R}{\mathrm{~d} \beta}>0$; if $\beta^{*}(k)>\frac{\mu \delta}{(\mu+\rho \sigma)(1+h(k))}$, $\frac{\mathrm{d} R}{\mathrm{~d} \beta}<0$. This implies that $R(\beta, k)$ is quasi-concave in $\beta$ and it reaches the maximum at $\beta^{*}(k)=\frac{\mu \delta}{(\mu+\rho \sigma)(1+h(k))}$, thus, we obtain equation (4.19).

$\alpha^{*}(k)$ is obtained by substituting the $\beta^{*}(k)$ into the equation (4.17), and the optimal investment level $z^{*}(k)$ is derived by substituting the $\beta^{*}(k)$ into equation (4.5), and to substitute the $\alpha^{*}(k), \beta^{*}(k)$ and $z^{*}(k)$ into the above $R(\beta, k)$, the maximum objective value $\mathrm{E}_{k}^{*}\left(\pi_{G}\right)$ could be calculated and satisfies equation (4.23). Therefore, this proposition is proved.

From the assumption $k Q_{0}(\mu+\rho \sigma) \beta \geq 1$, we have that $Q_{0}$ satisfies $Q_{0} \geq \frac{1+h(k)}{k \mu \delta}$.

Proposition 4.2 states that, the enterprise's final expected emission amount is $Q_{0} \mu e^{-k z}=\frac{1+h(k)}{k \delta}$, and the actual expected emission reduction is $Q_{0}\left(1-\mu e^{-k z}\right)=Q_{0}-\frac{1+h(k)}{k \delta}$. Equation (4.19) implies that, the enterprise obtains additional information rent $\int_{\underline{k}}^{k} \frac{\ln \left[\tau Q_{0}(\mu+\rho \sigma) \beta^{*}(\tau)\right]}{\tau^{2}} \mathrm{~d} \tau$ due to the private information, rather than the reservation utility $\underline{\pi}$.

We further obtained $\frac{\partial \mathrm{E}_{k}^{*}\left(\pi_{G}\right)}{\partial(\rho \sigma)}=-\frac{\mu}{k(\mu+\rho \sigma)^{2}}<0$, which means $\mathrm{E}_{k}^{*}\left(\pi_{G}\right)$ is decreasing in the enterprise's risk aversion $\rho$ and the actual variability of the stochastic emission $\sigma^{2}$, which is consistent with the traditional principal-agent theory model (see [11]).

\section{Two MONITORING MECHANISMS}

In this section, two monitoring mechanisms are proposed: the information-based, and the action-based. In the first stage, the government signs contracts with the supervisory authorities $I$ and designs the linear contract $(u, v)$, and then the supervisory authorities $I$ provides the same $(\alpha, \beta)$ for the enterprise. In the following subsections, these two monitoring mechanisms would be discussed separately.

\subsection{Information-based monitoring mechanism}

Under information-based monitoring mechanism, the government signs contracts with supervisory authorities $I$ for monitoring the enterprise's private technology information, the sequence of events is as follows: (1) The government and the supervisory authorities $I$ agree on signing a contract $(u, v) ;(2)$ The supervisory authorities $I$ decides whether to participate or not; (3) The supervisory authorities $I$ and the enterprise observe the actual technology level $k$; (4) After the agreement is signed, the supervisory authorities $I$ offers a contract $(\alpha, \beta)$ to the enterprise; (5) The enterprise decides whether to participate or not; (6) With the signed contract, the enterprise determines his unobservable investment level, and (7) The government and supervisory authorities $I$ offer incentive based on the realized emission amount. Since, the supervisory authorities $I$ also observes the technology level, she faces a genuine moral hazard problem while signing the contract with the enterprise.

Note that in this monitoring scenario, there exist two-stage principal-agent relationships, i.e., the relationship between the government and supervisory authorities $I$ as well as the relationship between supervisory authorities $I$ and the enterprise, and the intertwined upstream and downstream contracting issues. The government has to account for supervisory authorities $I$ and the enterprise's optimal decisions in the second stage when it formulates the contract to maximize its expected profit in the first stage. Here, backward induction would be used to solve the two-stage contract design problem.

To characterize the optimal monitoring mechanism, we start from the second stage in which supervisory authorities $I$ offer an optimal incentive contract $\left(\alpha_{I}^{*}, \beta_{I}^{*}\right)$ to induce the enterprise to invest optimal level. From 
equation (3.5), the enterprise chooses investment level and receives the expected utility as

$$
U_{I}(k \mid z)=\alpha-(\mu+\rho \sigma) Q_{0} \beta e^{-k z}-z .
$$

Maximizing the expected utility is equivalent to choosing the optimal investment level

$$
z_{I}^{*}=\arg \max _{z \geq 0} U_{I}(k \mid z)=\frac{\ln \left[k Q_{0}(\mu+\rho \sigma) \beta\right]}{k} .
$$

Substituting equation (5.2) into equation (5.1), the enterprise's optimal maximum utility is

$$
U_{I}(k)=\max _{z \geq 0} U_{I}(k \mid z)=\alpha-\frac{1+\ln \left[k Q_{0}(\mu+\rho \sigma) \beta\right]}{k} .
$$

Given the enterprise's optimal investment level, the optimal expected emission is $\mathrm{E}(Q)=\frac{\mu}{k(\mu+\rho \sigma) \beta}$, and consequently, supervisory authorities I's expected profit is given by

$$
\mathrm{E}\left(\pi_{I}\right)=u-\alpha-(v-\beta) \frac{\mu}{k(\mu+\rho \sigma) \beta} .
$$

Therefore, supervisory authorities I's goal is to find the contract that solves

$$
\begin{array}{ll} 
& \max _{\alpha, \beta} \mathrm{E}\left(\pi_{I}\right)=u-\alpha+\frac{\mu}{k(\mu+\rho \sigma)}\left(1-\frac{v}{\beta}\right), \\
\text { s.t. } & (\mathrm{IC}) U_{I}(k \mid z) \geq U_{I}\left(k \mid z^{\prime}\right), \\
& (\mathrm{IR}) U_{I}(k \mid z) \geq \underline{\pi} .
\end{array}
$$

The constraints (5.5) and (5.6) ensure enterprise's willingness to accept the contract. Equation (5.5) is IC constraint under moral hazard which ensures enterprise's full compliance. The IR constraint (5.6) ensures the participation of the enterprise. Lemma 5.1 summarizes the solution to the above problem.

Lemma 5.1. Given the contract $(u, v)$ and the technology level $k$, the supervisory authorities I optimally offers the incentive contract $\left(\alpha_{I}^{*}, \beta_{I}^{*}\right)$ as

$$
\alpha_{I}^{*}=\underline{\pi}+\frac{1+\ln \left(k \mu Q_{0} v\right)}{k}, \quad \beta_{I}^{*}=\frac{\mu v}{\mu+\rho \sigma},
$$

and induces the corresponding optimal investment level as

$$
z_{I}^{*}=\frac{\ln \left[k Q_{0}(\mu+\rho \sigma) \beta_{I}^{*}\right]}{k}=\frac{\ln \left(k \mu Q_{0} v\right)}{k},
$$

the supervisory authorities I receives an expected profit as

$$
\mathrm{E}^{*}\left(\pi_{I}\right)=u-\underline{\pi}-\frac{1+\ln \left(k \mu Q_{0} v\right)}{k}-\frac{\rho \sigma}{k(\mu+\rho \sigma)} .
$$

Proof. From equation (5.2), we can get that $z_{I}^{*}=\frac{\ln \left[k Q_{0}(\mu+\rho \sigma) \beta\right]}{k}$, and the binding IR constraint (5.6) leads to $\alpha_{I}=\underline{\pi}+\frac{1+\ln \left[k Q_{0}(\mu+\rho \sigma) \beta\right]}{k}$. Replace the $\alpha_{I}$ in the supervisory authorities $I$ 's objective function (5.4), the problem is reduced to

$$
\max _{\beta} \mathrm{E}\left(\pi_{I}\right)=u-\underline{\pi}-\frac{1+\ln \left[k Q_{0}(\mu+\rho \sigma) \beta\right]}{k}+\frac{\mu}{k(\mu+\rho \sigma)}\left(1-\frac{v}{\beta}\right) .
$$

Based on the first-order necessary condition $\frac{\partial \mathrm{E}\left(\pi_{I}\right)}{\partial \beta}=\frac{\mu v-(\mu+\rho \sigma) \beta}{k(\mu+\rho \sigma) \beta^{2}}$, obviously, $\mu v-(\mu+\rho \sigma) \beta$ is decreasing in $\beta$, we have 
If $\beta_{I}^{*}<\frac{\mu v}{(\mu+\rho \sigma)}, \frac{\partial \mathrm{E}\left(\pi_{I}\right)}{\partial \beta}>0 ; \beta_{I}^{*}>\frac{\mu v}{(\mu+\rho \sigma)}, \frac{\partial \mathrm{E}\left(\pi_{I}\right)}{\partial \beta}<0$. This implies that $\mathrm{E}\left(\pi_{I}\right)$ is quasi-concave in $\beta$ and it reaches the maximum at $\beta_{I}^{*}=\frac{\mu v}{(\mu+\rho \sigma)}$, thus, we obtain equation (5.7).

$\alpha_{I}^{*}$ is obtained by substituting the $\beta_{I}^{*}$ into equation (5.6), and the optimal investment level $z_{I}^{*}$ is derived by substituting the $\beta_{I}^{*}$ into equation (5.2), and to substitute the $\alpha_{I}^{*}, \beta_{I}^{*}$ and $z_{I}^{*}$ into the above $\mathrm{E}\left(\pi_{I}\right)$, the maximum objective value $\mathrm{E}\left(\pi_{I}\right)$ can be calculated as $\mathrm{E}^{*}\left(\pi_{I}\right)$ and hence it satisfies equation (5.9). Therefore, this lemma is proved.

After obtaining obtained the supervisory authorities I's optimal contract $\left(\alpha_{I}^{*}, \beta_{I}^{*}\right)$ for the enterprise, we proceed with the government's problem. The government would use $\left(\alpha_{I}^{*}, \beta_{I}^{*}\right)$ to formulate a contract $\left(u_{I}^{*}, v_{I}^{*}\right)$ for the supervisory authorities $I$ to choose, and to maximize her own expected profit.

Given the optimal investment level $z_{I}^{*}$ in Lemma 5.1, the optimal expected emission is $\frac{1}{k v}$, and consequently, the government's expected profit is given by

$$
\mathrm{E}\left(\pi_{G I}\right)=\mathrm{E}[B-u+(v-\delta) Q]=B-u+\frac{1}{k}\left(1-\frac{\delta}{v}\right) .
$$

Combining with supervisory authorities $I$ 's expected profit $\mathrm{E}^{*}\left(\pi_{I}\right)$, the government's goal is to find a suitable contract $\left(u_{I}^{*}, v_{I}^{*}\right)$, and the decision problem is expressed as follows

$$
\begin{aligned}
\max _{u, v} & \mathrm{E}_{k}\left(\pi_{G I}\right)=\mathrm{E}_{k}\left[B-u+\frac{1}{k}\left(1-\frac{\delta}{v}\right)\right], \\
& \text { s.t. (IR) } \mathrm{E}^{*}\left(\pi_{I}\right)=u-\underline{\pi}-\frac{1+\ln \left(k \mu Q_{0} v\right)}{k}-\frac{\rho \sigma}{k(\mu+\rho \sigma)} \geq \underline{I} .
\end{aligned}
$$

The IR constraint (5.12) ensures the participation of supervisory authorities $I$. The following proposition characterizes the optimal contracts offered by the government.

Proposition 5.2. Under information-based monitoring mechanism, the optimal incentive contract $\left(u_{I}^{*}, v_{I}^{*}\right)$ offered by the government is given by

$$
u_{I}^{*}=\underline{I}+\underline{\pi}+\frac{1+\ln \left(k \mu Q_{0} \delta\right)}{k}+\frac{\rho \sigma}{k(\mu+\rho \sigma)}, \quad v_{I}^{*}=\delta .
$$

Given the optimal incentive contract $\left(u_{I}^{*}, v_{I}^{*}\right)$, the corresponding optimal monitoring contract $\left(\alpha_{I}^{*}, \beta_{I}^{*}\right)$ offered by the supervisory authorities I is given by

$$
\alpha_{I}^{*}=\underline{\pi}+\frac{1+\ln \left(k \mu Q_{0} \delta\right)}{k}, \quad \beta_{I}^{*}=\frac{\mu \delta}{\mu+\rho \sigma} .
$$

The corresponding optimal investment level for the enterprise is given by

$$
z_{I}^{*}=\frac{\ln \left(k \mu Q_{0} \delta\right)}{k} .
$$

Under this contract, the government's expected payoff is

$$
\mathrm{E}_{k}^{*}\left(\pi_{G I}\right)=\mathrm{E}_{k}\left[B-\underline{I}-\underline{\pi}-\frac{1+\ln \left(k \mu Q_{0} \delta\right)}{k}-\frac{\rho \sigma}{k(\mu+\rho \sigma)}\right] .
$$

Proof. Due to binding constraint at optimality, $u$ could be replaced by equation (5.12) in the objective function (5.11) and reduce the problem into

$$
\max _{v} \mathrm{E}_{k}\left(\pi_{G I}\right)=\mathrm{E}_{k}\left[B-\underline{I}-\underline{\pi}-\frac{1+\ln \left(k \mu Q_{0} v\right)}{k}-\frac{\rho \sigma}{k(\mu+\rho \sigma)}+\frac{1}{k}\left(1-\frac{\delta}{v}\right)\right] .
$$


By the first-order condition $\frac{\partial \mathrm{E}\left(\pi_{G I}\right)}{\partial v}=\frac{\delta-v}{k v^{2}}$, it is obvious that, if $v_{I}^{*}<\delta, \frac{\partial \mathrm{E}\left(\pi_{G I}\right)}{\partial v}>0$, and if $v_{I}^{*}>\delta$, $\frac{\partial \mathrm{E}\left(\pi_{G I}\right)}{\partial v}<0$. This implies that the optimal commission rate is $v_{I}^{*}=\delta, u_{I}^{*}$ is obtained by substituting $v_{I}^{*}=\delta$ into the equation (5.12), and derivation of optimal investment level $z_{I}^{*}$ in equation (5.8), $\mathrm{E}_{k}^{*}\left(\pi_{G I}\right)$ could be calculated by substituting $\left(u_{I}^{*}, v_{I}^{*}\right)$. Hence, this proposition is proved.

It's relatively easy to verify that the optimal commission rate is greater than that in the benchmark setting of no monitoring, i.e., $\beta_{I}^{*}>\beta^{*}(k)$ holds. This implies that the supervisory authorities $I$ should set a higher incentive intensity to prevent non-compliance by the enterprise.

Under information-based monitoring mechanism, by equation (5.15), we can obtain the enterprise's final expected emission amount is $\frac{1}{k \delta}$, and the actual expected emission reduction is $Q_{0}-\frac{1}{k \delta}$.

Proposition 5.2 shows that the supervisory authorities $I$ 's profit margin is fixed at $v_{I}^{*}=\delta$. Compared to Proposition 4.2, under information-based monitoring mechanism, the optimal investment level is greater than that in the benchmark setting of no monitoring, the enterprise only obtains the reservation utility $U_{I}^{*}=\underline{\pi}$, instead of the additional information rent.

\subsection{Action-based monitoring mechanism}

Under action-based monitoring mechanism, the government signs contracts with supervisory authorities $A$ to monitor the enterprise's investment action. Hence, that supervisory authorities $A$ is able to observe the investment level and therefore could specify the required investment level in the contract. However, because of the unobservable technology level, a menu of contracts $(\alpha(k), \beta(k), z(k))$ should be offered for the enterprise to report $k$ truthfully, the sequence of events is as follows: (1) The government announces an incentive contract $(u, v)$ for supervisory authorities $A ;(2)$ The supervisory authorities $A$ decides whether to participate or not; (3) Under the signed contract, the enterprise observes the technology level; (4) The supervisory authorities $A$ offers the menu of contracts $(\alpha(k), \beta(k), z(k))$ to the enterprise; (5) The enterprise decides whether to participate or not; (6) Under the signed contract, the enterprise follows the unobservable investment level specified in the contract; and (7) Finally, the emission amount is realized and everyone receives the payoff according to the chosen contracts.

Using backward induction and starting with the enterprise's problem, in the second stage, we suppose that the enterprise had observed the technology level $k$ but had chosen the contract $\left(\alpha\left(k^{\prime}\right), \beta\left(k^{\prime}\right), z\left(k^{\prime}\right)\right)$ related to $k^{\prime}$, In this case, it would get $\alpha\left(k^{\prime}\right)-\beta\left(k^{\prime}\right) Q_{0} \varepsilon e^{-k z\left(k^{\prime}\right)}-z\left(k^{\prime}\right)$ as his net income and then the expected utility is given by

$$
U_{A}\left(k^{\prime} \mid k\right)=\alpha\left(k^{\prime}\right)-(\mu+\rho \sigma) \beta\left(k^{\prime}\right) Q_{0} e^{-k z\left(k^{\prime}\right)}-z\left(k^{\prime}\right),
$$

let $U_{A}(k)=U_{A}(k \mid k)$, thus $U_{A}(k)=\alpha(k)-(\mu+\rho \sigma) \beta(k) Q_{0} e^{-k z(k)}-z(k)$.

At equilibrium, the supervisory authorities $A$ would induce the enterprise to disclose his technology level $k$ truthfully by choosing the contract $(\alpha(k), \beta(k), z(k))$ followed by the expected emission amount $Q_{0} \mu e^{-k z(k)}$ and consequently the supervisory authorities $A$ 's expected profit is given by $\mathrm{E}\left(\pi_{A}\right)=u-\alpha(k)+(\beta(k)-v) Q_{0} \mu e^{-k z(k)}$. The supervisory authorities $A$ 's goal is to find the menu of contracts $(\alpha(k), \beta(k), z(k))$ that solves

$$
\begin{array}{ll} 
& \max _{\alpha(k), \beta(k), z(k)} \mathrm{E}_{k}\left(\pi_{A}\right)=\int_{\underline{k}}^{\bar{k}}\left[u-\alpha(k)+(\beta(k)-v) Q_{0} \mu e^{-k z(k)}\right] \mathrm{d} F(k), \\
\text { s.t. } & (\mathrm{TC}) U_{A}(k) \geq U_{A}\left(k^{\prime} \mid k\right), \\
& (\mathrm{IR}) U_{A}(k) \geq \underline{\pi} .
\end{array}
$$

The TC constraint (5.19) requires Truth-Telling and the IR constraint (5.20) guarantees the enterprise's participation. Lemma 5.3 summarizes the solution. 
Lemma 5.3. Given the incentive contract $(u, v)$, the optimal response $\left(\alpha_{A}^{*}(k), \beta_{A}^{*}(k)\right.$, $\left.z_{A}^{*}(k)\right)$ made by the supervisory authorities $A$ is given by

$$
\alpha_{A}^{*}(k)=\underline{\pi}+\frac{\ln \left(k \mu Q_{0} v\right)}{k}, \quad \beta_{A}^{*}(k)=0, \quad z_{A}^{*}(k)=\frac{\ln \left(k \mu Q_{0} v\right)}{k} .
$$

The supervisory authorities $A$ would receive an expected profit as

$$
\mathrm{E}_{k}^{*}\left(\pi_{A}\right)=\mathrm{E}_{k}\left[u-\underline{\pi}-\frac{1+\ln \left(k \mu Q_{0} v\right)}{k}\right] .
$$

Proof. It follows from the first-order necessary condition of the TC constraint that

$$
\frac{\mathrm{d} U_{A}(k)}{\mathrm{d} k}=(\mu+\rho \sigma) \beta(k) z(k) Q_{0} e^{-k z(k)} \geq 0,
$$

for all $k \in[\underline{k}, \bar{k}]$, which implies that $U_{A}(k)$ is increasing in $k$. The IR constraint (5.20) implies that $\min U_{A}(k)=U_{A}(\underline{k})=\underline{\pi}$, consequently,

$$
U_{A}(k)=\underline{\pi}+\int_{\underline{k}}^{k}(\mu+\rho \sigma) \beta(\tau) z(\tau) Q_{0} e^{-\tau z(\tau)} \mathrm{d} \tau,
$$

and the binding IR constraint leads to

$$
\alpha(k)=\underline{\pi}+z(k)+(\mu+\rho \sigma) \beta(k) Q_{0} e^{-k z(k)}+\int_{\underline{k}}^{k}(\mu+\rho \sigma) \beta(\tau) z(\tau) Q_{0} e^{-\tau z(\tau)} \mathrm{d} \tau .
$$

Replace the above $\alpha(k)$ in the supervisory authorities $A$ 's objective function (5.18), the supervisory authorities $A$ 's problem could be rewritten as (see the proof of Prop. 4.2)

$$
\begin{aligned}
\max _{\beta(k), z(k)} & \mathrm{E}_{k}\left[u-\underline{\pi}-z(k)-(\mu+\rho \sigma) \beta(k) Q_{0} e^{-k z(k)}\right. \\
- & \left.\int_{\underline{k}}^{k}(\mu+\rho \sigma) \beta(\tau) z(\tau) Q_{0} e^{-\tau z(\tau)} \mathrm{d} \tau+(\beta(k)-v) Q_{0} \mu e^{-k z(k)}\right] \\
= & \mathrm{E}_{k}\left[u-\underline{\pi}-z(k)-(\mu+\rho \sigma) \beta(k) Q_{0} e^{-k z(k)}\right. \\
& \left.-(\mu+\rho \sigma) \beta(k) z(k) Q_{0} e^{-k z(k)} H(k)+(\beta(k)-v) Q_{0} \mu e^{-k z(k)}\right] .
\end{aligned}
$$

Let $M(\beta)=u-\underline{\pi}-z(k)-(\mu+\rho \sigma) \beta(k) Q_{0} e^{-k z(k)}-(\mu+\rho \sigma) \beta(k) z(k) Q_{0} e^{-k z(k)} H(k)+(\beta(k)-v) Q_{0} \mu e^{-k z(k)}$, based on the first-order condition, $\frac{\mathrm{d} M}{\mathrm{~d} \beta}=-\rho \sigma Q_{0} e^{-k z(k)}-(\mu+\rho \sigma) z(k) Q_{0} e^{-k z(k)} H(k)<0,((H(k)>0)$, which implies $M$ is strictly decreasing in $\beta$, thus, the optimal commission rate is $\beta_{A}^{*}(k)=0$. We can get the corresponding optimal investment level is $z_{A}^{*}(k)=\frac{\ln \left(k \mu Q_{0} v\right)}{k} \cdot \alpha_{A}^{*}(k)$ would be obtained by replacing the $\beta_{A}^{*}(k)$, and the supervisory authorities $A$ 's maximum expected payoff $\mathrm{E}_{k}^{*}\left(\pi_{A}\right)$ is could be calculated. Therefore, this lemma is proved.

According to Lemma 5.3, the supervisory authorities $A$ should offer a fixed payment to the enterprise. As the supervisory authorities $A$ also observes the investment level, she faces adverse selection problem while contracting with the enterprise.

Having obtained the supervisory authorities $A$ 's optimal contract for the enterprise, we now proceed to consider the government's problem. The government should use $\left(\alpha_{A}^{*}(k), \beta_{A}^{*}(k), z_{A}^{*}(k)\right)$ to design the $\left(u_{A}^{*}, v_{A}^{*}\right)$ for 
the supervisory authorities $A$, and to maximize her own expected profit. Given the optimal investment level $z_{A}^{*}(k)$ in Lemma 5.3, the optimal expected emission is $\frac{1}{k v}$, and consequently the government's expected profit is $\mathrm{E}_{k}^{*}\left(\pi_{G A}\right)=\mathrm{E}_{k}\left[B-u+\frac{1}{k}\left(1-\frac{\delta}{v}\right)\right]$.

Combined with supervisory authorities $A$ 's expected profit $\mathrm{E}_{k}^{*}\left(\pi_{A}\right)$ in Lemma 5.3, the government's problem is to find the contract $\left(u_{A}^{*}, v_{A}^{*}\right)$ which could be expressed as follows

$$
\begin{aligned}
\max _{u, v} \mathrm{E}_{k}\left(\pi_{G I}\right) & =\mathrm{E}_{k}\left[B-u+\frac{1}{k}\left(1-\frac{\delta}{v}\right)\right], \\
\text { s.t. } \quad(\mathrm{IR}) \mathrm{E}_{k}^{*}\left(\pi_{A}\right) & =\mathrm{E}_{k}\left[u-\underline{\pi}-\frac{1+\ln \left(k \mu Q_{0} v\right)}{k}\right] \geq \underline{A} .
\end{aligned}
$$

The constraint (5.24) ensures the supervisory authorities $A$ 's participation. The following proposition characterizes the optimal contracts offered by the government and supervisory authorities $A$ under action-based monitoring mechanism.

Proposition 5.4. Under action-based monitoring mechanism, the optimal incentive contract $\left(u_{A}^{*}, v_{A}^{*}\right)$ offered by the government is given by

$$
u_{A}^{*}=\underline{\pi}+\underline{A}+\frac{1+\ln \left(k \mu Q_{0} \delta\right)}{k}, \quad v_{A}^{*}=\delta .
$$

Given the optimal incentive contract $\left(u_{A}^{*}, v_{A}^{*}\right)$, the corresponding optimal monitoring contract $\left(\alpha_{A}^{*}(k), \beta_{A}^{*}(k), z_{A}^{*}(k)\right)$ offered by the supervisory authorities $A$ is given by

$$
\alpha_{A}^{*}(k)=\underline{\pi}+\frac{1+\ln \left(k \mu Q_{0} \delta\right)}{k}, \quad \beta_{A}^{*}(k)=0, \quad z_{A}^{*}(k)=\frac{\ln \left(k \mu Q_{0} \delta\right)}{k} .
$$

Under this contract, the government's expected payoff is

$$
\mathrm{E}_{k}^{*}\left(\pi_{G A}\right)=\mathrm{E}_{k}\left[B-\underline{\pi}-\underline{A}-\frac{1+\ln \left(k \mu Q_{0} \delta\right)}{k}\right] .
$$

Proof. Observing at optimal level there is a binding constraint (5.24). Thus, the government's problem reduces to

$$
\max _{v} \mathrm{E}_{k}\left(\pi_{G A}\right)=\mathrm{E}_{k}\left[B-\underline{\pi}-\underline{A}-\frac{1+\ln \left(k \mu Q_{0} v\right)}{k}+\frac{1}{k}\left(1-\frac{\delta}{v}\right)\right] .
$$

By the first-order condition, the optimal commission rate is $v_{A}^{*}=\delta$, and $u_{A}^{*}$ is obtained by substituting $v_{A}^{*}=\delta$ into the equation (5.24), and the optimal investment level $z_{A}^{*}(k)$ is derived using equation $(5.21), \mathrm{E}_{k}^{*}\left(\pi_{G A}\right)$ could be calculated by substituting $\left(u_{A}^{*}, v_{A}^{*}\right)$. Hence, this proposition is proved.

Under action-based monitoring mechanism, we can further to obtain the enterprise's final expected emission amount is $\frac{1}{k \delta}$ according to $z_{A}^{*}(k)$, and the related expected emission reduction is $Q_{0}-\frac{1}{k \delta}$.

Proposition 5.4 states that, under action-based monitoring mechanism, the optimal investment level is also greater than that in the benchmark setting of no monitoring, and the enterprise only obtains the reservation utility $U_{A}^{*}=\underline{\pi}$, which is consistent with the information-based monitoring scenario.

\section{Analytical Results And COMPARison}

A salient problem faced by the government is, whether the government should contract with the enterprise directly, or hires the supervisory authorities to monitor the enterprise and whether there is a dominant monitoring mechanism? Now the results of two monitoring mechanisms would be compared with no monitoring scenario. 


\subsection{Comparisons}

According to Propositions 4.2, 5.2 and 5.4, we have

Proposition 6.1. When two monitoring mechanisms are compared with no monitoring scenario, the government's expected profit and the enterprise's expected utility and optimal investment level are related as

(1) $z_{A}^{*}(k)=z_{I}^{*} \geq z^{*}(k)$,

(2) $\mathrm{E}_{k}^{*}\left(\pi_{G A}\right) \geq \mathrm{E}_{k}^{*}\left(\pi_{G I}\right) \geq \mathrm{E}_{k}^{*}\left(\pi_{G}\right)$,

(3) $U_{E}^{*}(k) \geq U_{I}^{*}(k)=U_{A}^{*}(k)$.

Proof. Obviously, $\mathrm{E}_{k}^{*}\left(\pi_{G A}\right) \geq \mathrm{E}_{k}^{*}\left(\pi_{G I}\right)$ holds, $\mathrm{E}_{k}^{*}\left(\pi_{G I}\right)$ and $\mathrm{E}_{k}^{*}\left(\pi_{G}\right)$ are compared hence,

$$
k\left[\mathrm{E}_{k}^{*}\left(\pi_{G I}\right)-\mathrm{E}_{k}^{*}\left(\pi_{G}\right)\right]=\mathrm{E}_{k}\left[h(k) \ln \left(k Q_{0} \mu \delta\right)-(1+h(k)) \ln (1+h(k))+h(k)\right] .
$$

Let $\Psi(x)=x \ln \left(k Q_{0} \mu \delta\right)-(1+x) \ln (1+x)+x$, thus $\Psi^{\prime}(x)=\ln \left(k Q_{0} \mu \delta\right)-\ln (1+x) \geq 0$ (according to the Prop. 4.2, $z^{*}(k)=\frac{\ln \left[\frac{k Q_{0} \mu \delta}{1+h(k)}\right]}{k}$ and $\left.h(k)>0\right)$, which implies $\Psi(x)$ is increasing in $x \geq 0$, consequently, $\Psi(x) \geq \Psi(0)=0$ and $\mathrm{E}_{k}^{*}\left(\pi_{G I}\right) \geq \mathrm{E}_{k}^{*}\left(\pi_{G}\right)$ holds.

Obviously, $\frac{1}{k \delta}<\frac{1+h(k)}{k \delta}$ holds, which implies that the enterprise's final expected emission amount is less under monitoring mechanism, while on the other hand, the actual expected emission reduction is also higher under monitoring mechanism.

Proposition 6.1 states that the optimal investment level under monitoring mechanism for the enterprise is greater than its optimal value obtained in the scenario of no monitoring, which indicates that from the government's perspective, using both the information-based and action-based monitoring strategies could improve the enterprise's optimal investment level. The government's profit will increase under both the monitoring mechanism, with the action-based monitoring mechanism greatly improving the government's expected profit, thus implying that the action-based monitoring mechanism is more effective than the information-based monitoring mechanism in inducing better income. Proposition 6.1 also reveals that the enterprise no longer obtains additional information rent under monitoring mechanism.

\subsection{Numerical examples}

In this section, numerical studies have been conducted to complement the analytical findings in the previous sections by implementing the following base parameter set: $\mu=1, \sigma=2, \rho=1, \delta=4, \underline{\pi}=0, \underline{I}=0, \underline{A}=0$, $A=8, Q_{0}=e^{3}=20.08$. In addition, $k$ is uniformly distributed in $[1,3]$, i.e., $[\underline{k}, \bar{k}]=[1,3]$, thus $H(k)=3-k$, $h(k)=3 / k-1$. Based on our analytical results of previous sections, the optimal decisions under benchmark case and two monitoring scenarios could be calculated out.

From Proposition 4.2, for no monitoring scenario, from equations (4.19) to (4.23), the optimal of contract is:

$$
\alpha^{*}(k)=\int_{1}^{k} \frac{3+\ln 1.33+2 \ln \tau}{\tau^{2}} \mathrm{~d} \tau+\frac{4+\ln 1.33+2 \ln k}{k}, \quad \beta^{*}(k)=\frac{4 k}{9},
$$

while the enterprise's optimal investment level is:

$$
z^{*}(k)=\frac{3+\ln 1.33+2 \ln k}{k}=\frac{3.29+2 \ln k}{k},
$$

the enterprise's optimal expected utility is given by:

$$
\begin{aligned}
U_{E}^{*}(k) & =\int_{1}^{k} \frac{3+\ln 1.33+2 \ln \tau}{\tau^{2}} \mathrm{~d} \tau \\
& =5+\ln 1.33-\frac{5+\ln 1.33+2 \ln k}{k}=5.29-\frac{5.29+2 \ln k}{k},
\end{aligned}
$$


and the government's optimal expected profit $\mathrm{E}_{k}^{*}\left(\pi_{G}\right)$ satisfies

$$
\pi_{G}=8-\frac{2}{3 k}-\frac{3(4+\ln 1.33+2 \ln k)}{k^{2}}=8-\frac{12.86+6 \ln k+0.67 k}{k^{2}} .
$$

According to Proposition 5.2, if the the government adopts information-based monitoring mechanism, the optimal monitoring contract for the enterprise is as follows $\alpha_{I}^{*}=\frac{5.39+\ln k}{k}, \beta_{I}^{*}=1.33$, the enterprise's optimal investment level is $z_{I}^{*}=\frac{4.39+\ln k}{k}$, and the government's expected optimal profit is $\mathrm{E}_{k}^{*}\left(\pi_{G I}\right)=\mathrm{E}_{k}\left[8-\frac{6.06+\ln k}{k}\right]$.

If the government adopts action-based monitoring mechanism, the corresponding optimal monitoring menu contract offered by the supervisory authorities $A$ is given by $\alpha_{A}^{*}(k)=\frac{5.39+\ln k}{k}, \beta_{A}^{*}(k)=0, z_{A}^{*}(k)=\frac{4.39+\ln k}{k}$, and the government's expected profit is $\mathrm{E}_{k}^{*}\left(\pi_{G A}\right)=\mathrm{E}_{k}\left[8-\frac{5.39+\ln k}{k}\right]$.

Thus $\mathrm{E}_{k}^{*}\left(\pi_{G A}\right) \geq \mathrm{E}_{k}^{*}\left(\pi_{G I}\right) \geq \mathrm{E}_{k}^{*}\left(\pi_{G}\right), z_{A}^{*}(k)=z_{I}^{*} \geq z^{*}(k), \beta_{I}^{*}>\beta^{*}(k)$ and $U_{E}^{*}(k)>0$ hold.

\section{Conclusions}

Governments across the globe are developing regulatory instruments to reduce Greenhouse gases emissions, as well as bridge support for low-carbon technologies. In this article, two monitoring mechanisms were proposed for the government to induce emission reduction under dual asymmetric information: the information-based and action-based monitoring mechanism. Both the enterprise's investment for emission reduction and the technology level are assumed to be his private information. A two stage principal-agent monitoring model was presented followed by derivation of the respective optimal incentive contracts to examine the impact of monitoring mechanisms on incentive contracts. Furthermore, the corresponding optimal monitoring strategies were analyzed and compared with the no monitoring scenario. The results verify the effectiveness of hiring the supervisory authorities to monitor the enterprise's emission reduction with the action-based monitoring strategy being more effective. Under both the monitoring mechanisms, both the government's expected payoff and emission reduction would increase, being even higher under the action-based monitoring mechanism. The enterprise would not obtain the additional information rent and make additional investment on emission reduction. Numerical studies were conducted to understand the proposed models and validate the propositions.

Firstly for the sake of simplicity, the mean-variance method was adopted to measure the enterprise's risk concerns. In future research, the widely used negative exponential utility function, or the conditional valueat-risk (CVaR) criterion would be cited as the enterprise's risk measure (see [3]), followed by investigation of influence of two types of monitoring mechanisms on decisions and utility of all stake holders. Secondly, this paper assumes that the enterprise depicts risk-averse attitude. If the degree of risk aversion is the enterprise's private information, in reality, the enterprise might pretend to be more risk averse to induce the government to err in incentive, which may yield interesting insights. Finally, only the traditional linear compensation contract and fully rational players have been considered, the quota-based compensation plan (see [15]) and the behavioral characteristics model (see $[6,18]$ ) should also be investigated. Future work could include consideration of the emission reductions among multiple stakeholders simultaneously, including the central governments, local governments, enterprise, supervisory authorities, retailers, residents and the consumers, and extending the primal model to more complex monitoring settings (see [10]).

Acknowledgements. The authors are grateful for the financial support provided by the Think Tank of Energy Mining Economy (2018 Project for Cultural Evolution and Creation of CUMT 2018WHCC01).

\section{REFERENCES}

[1] C.E. Bai, Earnings profile and monitoring under asymmetric information. Econ. Lett. 57 (1997) 219-225.

[2] D.P. Baron and D. Besanko, Monitoring, moral hazard, asymmetric information, and risk sharing in procurement contracting. Rand J. Econ. 18 (1987) 509-532.

[3] Y. Chen, M. Xu and Z.G. Zhang, Technical Note - A risk-averse newsvendor model under the CVaR criterion. Oper. Res. 57 (2009) 1040-1044. 
[4] C. Cheng, M. Qi, X. Wang and Y. Zhang, Multi-period inventory routing problem under carbon emission regulations. Int. J. Prod. Econ. 182 (2016) 263-275.

[5] T.M. Choi, D. Li and H. Yan, Mean-variance analysis of a single supplier and retailer supply chain under a returns policy. Eur. J. Oper. Res. 184 (2008) 356-376.

[6] A.B. da Silva Rocha and G.M. Salomão, Environmental policy regulation and corporate compliance in evolutionary game models with well-mixed and structured populations. Eur. J. Oper. Res. 279 (2019) 486-501.

[7] D. Demougin and C. Fluet, Monitoring versus incentives. Eur. Econ. Rev. 45 (2001) 1741-1764.

[8] S. Du, L. Zhu, L. Liang and F. Ma, Emission-dependent supply chain and environment-policy-making in the "cap-and-trade" system. Energy Policy 57 (2013) 61-67.

[9] M. Huang, J. Tu, X. Chao and D. Jin, Quality risk in logistics outsourcing: a fourth party logistics perspective. Eur. J. Oper. Res. 276 (2019) 855-879.

[10] S. Huddart and P.J. Liang, Profit sharing and monitoring in partnerships. J. Accounting Econ. 40 (2005) $153-187$.

[11] B. Holmstrom and P. Milgrom, Aggregation and linearity in the provision of intertemporal incentives. Econometrica 55 (1985) 303-328.

[12] K. Joseph and A. Thevaranjian, Monitoring and incentives in sales organizations: An agency-theoretic perspective. Marketing Sci. 17 (1998) 107-123.

[13] L.C. Kung and Y.J. Chen, Monitoring the market or the salesperson? The value of information in a multilayer supply chain. Naval Res. Logistics 58 (2011) 743-762.

[14] H.S. Lau and H.L. Lau, Manufacturer's pricing strategy and return policy for a single-period commodity. Eur. J. Oper. Res. 116 (1999) 291-304.

[15] C.Y. Lee and R. Yang, Compensation plan for competing salespersons under asymmetric information. Eur. J. Oper. Res. 227 (2013) 570-580.

[16] Q. Li, B. Li, P. Chen and P. Hou, Dual-channel supply chain decisions under asymmetric information with a risk-averse retailer. Ann. Oper. Res. 257 (2015) 423-447.

[17] I.A. Mackenzie and M. Ohndorf, Optimal monitoring for project-based emissions trading systems under asymmetric information. J. Regul. Econ. 42 (2012) 180-203.

[18] S. Panda, N.M. Modak, M. Basu and S.K. Goyal, Channel coordination and profit distribution in a social responsible three-layer supply chain. Int. J. Prod. Econ. 168 (2015) 224-233.

[19] J. Sheng, U.A. Ozturk and S. Zhang, Effects of asymmetric information and reference emission levels on the emissions from deforestation and degradation. J. Cleaner Prod. 133 (2016) 1118-1127.

[20] B. Wu, P. Liu and X. Xu, An evolutionary analysis of low-carbon strategies based on the government-enterprise game in the complex network context. J. Cleaner Prod. 141 (2017) 168-179.

[21] G. Xu, B. Dan, X. Zhang and C. Liu, Coordinating a dual-channel supply chain with risk-averse under a two-way revenue sharing contract. Int. J. Prod. Econ. 147 (2014) 171-179.

[22] K. Yang, Y. Lan and R. Zhao, Monitoring mechanisms in new product development with risk-averse project manager. J. Intell. Manuf. 28 (2017) 667-681. 